\title{
Community hospital experience using electromagnetic navigation bronchoscopy system integrating tidal volume computed tomography mapping
}

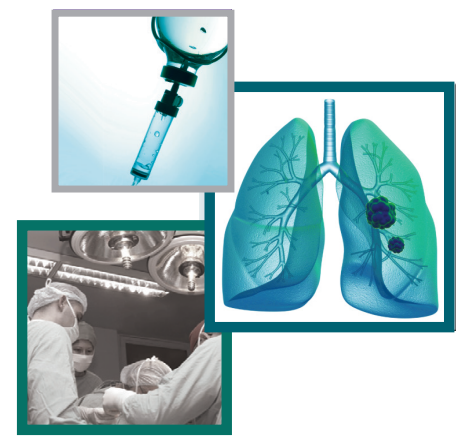

\author{
Abhijit A Raval' \& Leah Amir ${ }^{*, 2}$
}

\section{Practice points}

- The community pulmonologist will be faced with higher volumes of patients requiring a confirmatory diagnosis for positive lung computed tomography.

- Detecting lung cancer early requires the least minimally invasive method having the lowest risk profile and the highest diagnostic yield.

- Patients and the institution's tumor review board will need to understand minimally invasive techniques and their value to the patient.

- Electromagnetic navigation using tidal volume computed tomography mapping has a high diagnostic yield with a low-risk profile enabling minimally invasive lung tissue biopsy.

Results of the first 50 consecutive patients referred for bronchoscopy or surgery by the tumor review board to confirm suspect lung lesions identified by computed tomography. Electromagnetic navigation was used to biopsy peripheral pulmonary nodules, $(19.3 \pm 10.7 \mathrm{~mm})$. An electromagnetic tracking system was used to detect miniature position sensors integrated directly into tip-tracked instruments advanced through a $2 \mathrm{~mm}$ working channel in a bronchoscope. Learning curve, diagnostic yield, safety and use of the 4D positional information on the patient's tidal volume expiration computed tomography map demonstrate a potential to improve the diagnostic yield of transbronchial biopsies of peripheral pulmonary nodules less than $30 \mathrm{~mm}$ reporting a diagnostic yield of $83.3 \%(40 / 48)$. Early experience was safe and effective, with a limited learning curve.

First draft submitted: 17 November 2015; Accepted for publication: 22 March 2016; Published online: 8 April 2016

Lung cancer accounts for approximately $27 \%$ of all cancer deaths and is by far the leading cause of cancer death among both men and women. Each year, more people die of lung cancer than of colon, breast and prostate cancers combined. Lung cancer surpasses breast cancer as the biggest cancer killer of women. The American Cancer Society expects 221,200 new cases (115,610 in men and 105,590 in women) with 158,040 deaths from lung cancer, (86,380 in men and 71,660 women) in 2015 [1]. The United States Prevention Task Force (USPSTF) recommends high-risk patients should be screened for the presence of lung cancer using low-dose computed tomography (LDCT) to aid earlier detection. Earlier detection is poised to reduce patient morbidity and save lives yet is likely to increase the number of smaller distal pulmonary lesions requiring biopsy or surgery [2].

The National Lung Screening Trial (NLST) demonstrated a 20\% reduction in lung cancer mortality for the LDCT screening arm, thus recommending screening for high-risk patients. However,

\section{KEYWORDS}

- bronchoscopy

- electromagnetic

navigation $\bullet$ tidal volume expiration 
$96.4 \%$ of the NLST patients who today would meet the USPSTF lung cancer screening criteria had false positives [3]. This high number of patients suitable for lung cancer screening creates a diagnostic challenge for the community pulmonologist who must become proficient using a safe and effective diagnostic method to identify the positive peripheral pulmonary nodule (PPN) while avoiding costly procedures that expose patients to increased morbidity. While watchful waiting is suitable for the smallest lung lesions, Fleischner Society guidelines recommend biopsying lesions that are greater than $8 \mathrm{~mm}$ in this high-risk population. NLST found $39.1 \%$ patients had at least one positive screening result, compared with $16 \%$ positive results in the chest radiograph arm. A positive screening result was defined as at least one nodule $\geq 4 \mathrm{~mm}$ in diameter. Out of the positive LDCT screened patients, 1638 patients were recommended for a follow-up percutaneous computer tomography (CT)-guided transthoracic needle aspiration (TTNA) examination, bronchoscopy or a surgical procedure resulting in malignancy findings of $39.6 \%(649)$ [3].

\section{Clinical significance \& rationale}

Screening high-risk patients, absence of symptoms associated with advanced stages of lung cancer, ushers in a new patient management paradigm. Patients with positive findings will be referred for a confirmatory diagnosis. The community pulmonologist in collaboration with the primary care physician (PCP) and their institutions tumor review board must assist patients in making informed decisions regarding lung biopsy options. A lung biopsy method with higher specificity to rule out the presence of lung cancer with a low complication profile offers a cost-effective solution to the healthcare system and to the patient. This high volume of nonsymptomatic yet high-risk patients is likely to have a low incidence of positive lung cancer findings thus commanding a biopsy method with a low incidence patient complications to access and biopsy PPN $<30 \mathrm{~mm}$.

The presence of benign lesions in $40-60 \%$ of solitary pulmonary nodules surgically removed signifies a need for a less invasive approach, able to preserve lung tissue and reduce patient morbidity [4]. A finding of benign tissue in the lung after a positive CT impacts patient management. With the increase in positive CT findings less invasive biopsy methods are required to verify the diagnosis yet reduce the increased patient morbidity and costs of a population recommended for verification of suspect lung cancer. A lung biopsy result indicating the suspect lung lesion is benign is by definition not malignant. A benign tumor is not expected to spread to other parts of the body. They may grow slowly, or might even stop growing or shrink. Typically benign lung lesions are usually not lifethreatening, and they do not generally need to be removed. A benign lung lesion could expand and push against nearby tissues but it will not invade, destroy or replace other tissues. Consideration for safe, effective patient management must be given to aid clinical decision-making for highrisk patients having co-morbidities and pulmonary disease who are referred for follow-up resulting from a suspect lung lesion found on LDCT. Some of these patients may be poor surgical candidates; therefore a less invasive method is sought.

For the community pulmonologist and their patient, selecting the most clinically appropriate biopsy technique for PPNs presents a difficult clinical complication/benefit decision. Radiographic findings including CT of nodule size, location, nodule characteristics, patient co-morbidities, patient respiratory function, patient significant history and postprocedure patient management are all carefully weighted by the pulmonologist, the patient, the institutional tumor review board and the patient's PCP. Balancing all these factors, the lung team strives to determine the patient's specific risk/benefit profile to select the most appropriate, outcome orientated treatment plan to achieve a definitive diagnosis that will guide patient management. It is imperative that definitive diagnosis be completed on these patients.

A positive CT finding follows standard practice guidelines to aid diagnosis and patient management [5]. There are three well-accepted patient management regimens based on the patient's probability of malignancy. Watchful waiting with serial CT scans for lesions less than $8 \mathrm{~mm}$, tissue biopsy for pathologic diagnosis and direct surgical excision. When including the patient's wishes, they may request even a lesion less than $8 \mathrm{~mm}$ to be biopsied. Watchful waiting for lesions greater than $8 \mathrm{~mm}$ is not consistent with the USPSTF intent to identify and treat lung cancer at stage I or stage II. This approach mitigates the significant benefit intended of earlier detection for lung cancer. If 
lung tissue biopsy is the selected option, then CT-guided TTNA is preferred. It has a high diagnostic yield slightly below $90 \%$, yet a pneumothorax rate of about $30 \%$, of which $15 \%$ of the patient's tend to require a chest tube. TTNA represents a high patient morbidity profile [6-9]. Flexible bronchoscopy is another diagnostic option due to the lower pneumothorax rate, however, the sensitivity of traditional bronchoscopic biopsy is only $34 \%$ for nodules less than $20 \mathrm{~mm}$, and as low as $14 \%$ for the PPN $[7,10]$. The sensitivity increases to $63 \%$ when nodules are greater than $20 \mathrm{~cm}$ in size, but decreases as the distance from the hilum increases $[10,11]$. A lesion having a bronchus sign leading to the lesion increases the success of transbronchial biopsy and brushing with a diagnostic yield approaching 70\% [12].

Increased complications of TTNA or surgical resection are costly to the healthcare provider while causing the patient to suffer undue morbidity. Screening the higher volume population with a reduced risk for a positive finding requires careful clinical decision-making by the physician, their patient and the tumor review board. Methods enabling a diagnosis of PPN less than $30 \mathrm{~mm}$ are appropriate for this patient population due to their low-risk profile. The cost-effectiveness of electromagnetic navigation (EMN) procedures has been compared with CT-guided TTNA procedures. EMN procedures have demonstrated a significant cost reduction to the hospital, especially with the addition of EMN-TTNA capabilities in the bronchoscopy suite [13]. Tidal volume expiration (TV-EXP)-mapped EMN capturing breath motion during the procedure offers an opportunity to improve on accessing the PPN and to reduce the incidence of complications.

\section{Aims of the study}

Provide minimally invasive solution for patients recommended for biopsy or surgery as recommended by the multidisciplinary hospital tumor review board for follow-up due to a positive CT diagnostic evaluation and a thorough analysis of each patient's clinical findings and medical history. The primary objective was to measure the total diagnostic yield relative to location and size of the lung lesion when using the TV-EXP-mapped EMN system for the first 50 consecutive patients (61 lesions) in the absence of radial-endobronchial ultrasound (EBUS), fluoroscopic navigation or rapid on-site cytology (ROSE). Additionally, we are reporting on the overall performance of the TV-EXPmapped EMN system; community pulmonologist learning curve of the TV-EXP-mapped EMN technology; safety of the TV-EXPmapped EMN system; and ultimately the clinical appropriateness of TV-EXP-mapped EMN to comply with the decision-making needs for the community pulmonologist receiving a high volume of patients referred for further evaluation due to a positive CT. Overall diagnostic yield and bronchus sign for TV-EXP-mapped EMN, complications, and patient follow-up are reported. Including in the findings TV-EXPmapped EMN is compared with pulmonary nodules that were previously sent routinely for TTNA to measure the accuracy of a rule out for lung cancer.

\section{Materials \& methods}

\section{- TV-EXP to remove lung motion artifact} limitations

Pulmonologists and thoracic surgeons are challenged to effectively access PPNs that do not have an airway leading directly to their position. Over the past several years, total lung capacity (TLC)-mapped electromagnetic navigation bronchoscopy (ENB) has proven effective at being able to assess some pulmonary nodules; however, the lung lesions were more centrally located, rather than the smaller, more peripheral pulmonary nodules expected in the lung cancer screening population. TV-EXP-mapped EMN systems use hybrid imaging protocols and multiple access techniques to improve diagnostic yields for these challenging PPNs.

TLC-mapped ENB systems in conjunction with radial-EBUS, fluoroscopic navigation and ROSE have been reported in the peer-reviewed literature, however, overall their standard diagnostic yield ranges $50-70 \%$ [9,11-12]. Different from the TLC-mapped ENB systems, TV-EXPmapped EMN systems use hybrid imaging protocols, respiratory gating, electromagnetic (EM) tip tracked instruments and multiple approaches (endobronchial and percutaneous) providing an opportunity to adapt this technology in the diagnosis of lung cancer at earlier stages. TLCmapped ENB systems with radial-EBUS offer a less invasive option but improvement is needed beyond their diagnostic yield.

The malignancy rate for symptomatic patients is $70 \%$ or higher with lung cancer at stage III or IV ensuring an $80 \%$ death rate at 5 years. 
This is significantly higher than $<4 \%$ incidence found in the nonsymptomatic screening patients in the NLST. Patients meeting the USPSTF criteria may likely not have evident symptoms and therefore are likely to have a lower probability of positive findings greater than $4 \%$ but certainly less than $70 \%$. Clearly patients with a probable lower rate of malignancy require a less invasive solution to identify the suspect lesion relative to cancer or a different tissue abnormality. Physicians will be challenged to determine which patients are better served with an invasive procedure TTNA or transthoracic needle biopsy (TTNB), or if indeed the less invasive TV-EXP-mapped EMN can provide a suitable diagnostic yield given the risk/benefit profile of the individual patient.

\section{Points to consider when selecting the optimal method}

- TLC-mapped ENB systems may not be well suited for PPNs

Eberhardt [14] delineated TLC-mapped ENB yield from radial EBUS, reporting 59\% yield for ENB for lesions $28.0 \pm 8 \mathrm{~mm}$. The diagnostic yield of lower lobes was $29 \%$. It was hypothesized that the reduced yield rate could be due to using CT images acquired in only a single breath hold measured as TLC and therefore not correctly compensating for respiratory movement.

Nabavizadeh [15] used a TLC-mapped ENB system in conjunction with radial EBUS and fluoroscopic guidance reporting a diagnostic yield of $32 \%$. During the procedure fiducials were placed. They were found to be $10-50 \mathrm{~mm}$ from the lesion surface in $27 \%$ of the patients, therefore providing further evidence that TLCmapped ENB may not be suitable to access and biopsy of PPN.

Becker [16], Schwarz [17] and Gildea [18] used a TLC-mapped ENB system in conjunction with fluoroscopic guidance to achieve a diagnostic yield of 69, 69 and 73\%, respectively, with an average lesion size of $33.5 \pm 11 \mathrm{~mm}$. These findings do not delineate between ENB and fluoroscopic guidance which contributed to the overall yield. Data regarding the mean distance from the lesion center measuring $9 \pm 5 \mathrm{~mm}$ could be at least $2-23 \mathrm{~mm}$ from the center of the lesion. This evidence suggests TLC-mapped ENB may not be the suitable option to access and biopsy the PPNs. Please refer to Table 1 for a breakdown of the data.

\section{- Study design}

The first consecutive 50 patients referred to a community pulmonologist for suspect lung cancer diagnostic follow-up resulting from a positive CT were treated from July 2012 to May 2013. Each referred patient's clinical findings; medical history and risk for lung cancer were first screened by the hospital tumor review board. The tumor review board comprised pulmonologists, radiologists, oncologists and internal medicine physicians, who studied each patient's overall medical history, family history, signs and symptoms, co-morbidities, smoking history and recent $\mathrm{CT}$ findings to arrive at the unanimous conclusion that the patient's evaluation would benefit from a lung tissue biopsy to confirm the presence or absence of suspect lung cancer. In the combined clinical judgement of the hospital tumor review board, the patient was recommended for lung biopsy. All of the patients had a positive CT scan showing a suspect lung lesion. In light of the USPSTF lung cancer screening guidelines, combined with the patient's clinical evaluation, and recommendation of the tumor review board, these patients were treated by the community pulmonologist. Each patient was consented to allow the use of the TV-EXP EMN procedure along with linear-EBUS as needed compliant with the recommendations of the American College of Chest Physicians [6,19]. The CT was read by the pulmonologist to aid in the TV-EXP EMN technique.

Patients were followed for 24 months to compare long-term outcomes with initial findings. Data were collected using retrospective chart review. The 50 consecutive patients underwent diagnostic TV-EXP-mapped EMN using the SPiNDrive System ${ }^{\mathrm{TM}}$ (Veran Medical Technologies MO, USA). Under IRB approval and with patient's consent each patient's, TV-EXP-mapped EMN procedure time and experience, diagnostic test reports and followup treatment and findings were extracted and included in an excel spreadsheet (Version 15) for analysis. Biopsy findings and long-term follow-up were reported for diagnostic yield and the TV-EXP-mapped EMN performance was reported for patient's safety, pulmonologist learning curve and contribution to overall diagnostic yield. The final diagnosis was determined by: repeat CT, or as recommended by the pulmonologist or tumor review board, these patients were referred to TTNA, or lung resection and biopsy. 
Table 1. Decision criteria for diagnostic yield of peripheral small lung lesions.

\begin{tabular}{|c|c|c|c|c|c|}
\hline Study (year) & $\begin{array}{l}\text { Diagnostic } \\
\text { yield (\%) }\end{array}$ & $\begin{array}{l}\text { Lesion size } \\
(\mathrm{mm})\end{array}$ & $\begin{array}{l}\text { Patients } \\
\text { evaluated (n) }\end{array}$ & Method & Ref. \\
\hline $\begin{array}{l}\text { Eberhardt et al. } \\
\text { (2007) }\end{array}$ & 59 & $28 \pm 8$ & 118 & ENB & [14] \\
\hline $\begin{array}{l}\text { Eberhardt et al. } \\
\text { (2007) }\end{array}$ & 69 & $25 \pm 5$ & 118 & EBUS & [14] \\
\hline $\begin{array}{l}\text { Nabavizadeh et al. } \\
\text { (2014) }\end{array}$ & 32 & $\begin{array}{l}22.7 \\
\text { (range: 8-44) }\end{array}$ & $\begin{array}{l}31 \text { patients, } \\
105 \text { fiducials } \\
\text { placed }\end{array}$ & ENB with daily $\mathrm{CBCT}$ & [15] \\
\hline Becker et al. (2005) & 69 & $24 \pm 5.4$ & 29 & $\begin{array}{l}\text { ENB conventional breath } \\
\text { hold }\end{array}$ & [16] \\
\hline $\begin{array}{l}\text { Schwarz et al. } \\
\text { (2006) }\end{array}$ & 69 & $33.5 \pm 11$ & 13 & ENB & [17] \\
\hline Gildea et al. (2006) & 73 & $22.8 \pm 12.6$ & 60 & ENB & [18] \\
\hline
\end{tabular}

\section{- Patient population \& lung lesions}

The study population was representative of patients having a positive finding on a screening $\mathrm{CT}$ who were also found to be at high risk for lung cancer by the hospital tumor review board. There was no statistically significant difference among the study population of 28 males and 22 females ranging in age from 31 to 87 years with an average age of $67.7 \pm 12.2$ reported in Table 2. The average size of the lesion was $19.3 \pm 10.7 \mathrm{~mm}$, ranging from 4 to $49 \mathrm{~mm}$. There were a total of 61 lesions biopsied in the 50 patients. Lesion location was mapped and related to diagnostic yield.

A clear airway was confirmed using flexible bronchoscopy (Olympus America, Center Valley, PA, USA). All procedures were performed using a conscious sedation protocol including intravenous versed (midazolam-HCl: $6 \mathrm{mg}$ ) and fentanyl $(75-125 \mu \mathrm{g})$ provided as needed at the discretion of the anesthesiologist. Local anesthesia comprised topical lidocaine in the oropharynx and airways as required throughout the procedure. Fluoroscopic guidance was not utilized. It is not required for TV-EXP-mapped EMN. TV-EXP-mapped EMN using EM tip-tracked forceps was used after linear EBUS (Olympus America, PA, USA) sampling of the lymph nodes of the main carina to immediately determine the stage of lung cancer. If a lung cancer stage is determined no further biopsies are needed, however if the initial staging is negative further biopsies are required to verify the absence of lung cancer. TV-EXP-mapped EMN using EM tiptracked forces sampled 48 remaining patients.
Biopsied tissue samples were sent to the hospital pathology laboratory for further diagnosis.

\section{- TV-EXP-mapped EMN technique}

The SPiNDrive system using a hybrid inspirationexpiration CT scan pair creates a TV-EXP CT map for navigation. EMN systems use electromagnetic localization systems to guide the pulmonologist through the patient's airways to the lung nodule of interest. Localization is based on maps created from CT images. In EMN procedures, the CT scan information is commonly used for three purposes: to map the pathway to the target/nodule, registration and the display of navigated instruments.

The hybrid inspiration-expiration CT scan follows a scanning protocol that uses low-dose parameters to acquire a scan pair for EMN procedures. Patients are frequently closer to a tidal breathing expiration state during the procedure rather than a TLC arms-up position. The objective of the TV-EXP process is to have the optimal CT map for accurate EMN procedures. The pulmonologist uses the system to identify optimal airway segmentation, target/nodule planning and finally use deformation algorithm maps to a tidal breathing expiration scan [21]. The TV-EXP process better accommodates to allow the pulmonologist to consistently reach targets less than $20 \mathrm{~mm}$ in size by compensating for respiratory movement. The use of referral, TLC only, scans may correlate to lower accuracy due to the patient state during the procedure not matching the patient state during the acquisition of the CT utilized to create the navigation map [22]. 
Table 2. Characteristics of the study population.

\begin{tabular}{ll} 
Characteristics & TV-EXP-mapped EMN \\
Number of patients & $48^{\dagger}$ \\
Female gender & 21 \\
Male gender & 27 \\
Age, mean \pm standard deviation; years & $67.7 \pm 12.2$ \\
Type of sedation: & \\
- Moderate/conscious sedation & 48 \\
- General anesthesia & 0 \\
- Number of lesions biopsied & 61 \\
- Size, mean \pm standard deviation; mm & $19.3 \pm 10.7$ \\
- Number of benign lesions & 29 \\
- Number of malignant lesions & 19 \\
Distribution of lesion size by number of lesions: & \\
- $\leq 10$ mm & 15 \\
- $\leq 20$ mm & 22 \\
- 21-30 mm & 14 \\
- >30 mm & 10 \\
Number of lesions and lobar location: & \\
- Right upper lobe & 23 \\
- Right middle lobe & 3 \\
- Right lower lobe & 16 \\
- Left upper lobe & 11 \\
- Left lower lobe & 8 \\
\hline †5 patients were consecutively treated; however, two patients did not progress to the TV-EXP-mapped EMN \\
procedure due to excessive coughing during linear endobronchial ultrasound. \\
EMN: Electromagnetic navigation; TV-EXP: Tidal volume expiration.
\end{tabular}

The differences between TV and TLC on accuracy of locating a PPN are critical to achieve a more representative location of the PPN. Registration is the process of matching a CT image space to patient lesion space. The SPiNDrive system uses a fiducial patient tracker enabling respiratory gating and computer or automatic registration of the patient requiring no user interaction. Conversely, TLC-mapped ENB systems use lumen registration to match the physical 3D state of the patient near expiration to a segmented airway from a TLC inspiration referral scan. Multiple registration error components may contribute to the inaccuracy of lumen registration such as user error from over or under sampling during point cloud collection, no respiratory gating, and the physical difference between the patient and CT scan due to arm position and respiratory state. By using the TV-EXP mapping EMN procedure these inherent inaccuracies of TLC-mapped ENB are corrected.

Registration accuracy of TLC-mapped ENB has been reported to be $2.6 \pm 1.1 \mathrm{~mm}$ in $\mathrm{CT}$ phantom testing [23] and average fiducial target registration error (AFTRE) of $0.3-0.5 \mathrm{~mm}$. A meta-analysis including 15 ENB studies, all with greater than ten patients, totaling 1033 lung nodules, found the discordance between the virtual bronchoscopic landmarks using TLCmapped ENB and the actual anatomic reference points (e.g., the main carina and lobar carinas) evaluated by AFTRE was a median of $4.6 \mathrm{~mm}$ (range: $3.6-6.6 \mathrm{~mm}$ ), with an overall pooled AFTRE of $5.1 \mathrm{~mm}$ [20].

\section{- TV-EXP mapping EMN navigation procedure}

Using the SPiNDrive, EM sensors were integrated directly into $1.8-1.9 \mathrm{~mm}$ sampling devices, or forceps used in this study, allowing tracking to be maintained at all times. Figure 1 below illustrates a typical navigation screen using the EM sensors of the SPiNDrive system. Axial, Coronal and Oblique CT cut planes are provided as well as $3 \mathrm{D}$ rendered airway views from the perspective of flying through or flying above the airway. Additionally, this EM tip tracking technique provides continuous patient tracking and respiratory gating that is displayed in the lower left quadrant of the screen.

\section{- Patient confirmatory diagnosis \& follow-up}

The community hospital multidisciplinary tumor review board considered many variables when deciding on the diagnostic evaluation for each of the patients with a positive CT finding. Patient's wishes, overall lung function, medical and family history, the patient's risk factors for invasive procedures, including any pre-existing conditions promulgating lung cancer were all considered before concluding on the optimal approach for each individual patient. Patient's with an initial biopsy result reported from pathology as negative for malignancy continued to be followed using either an additional diagnostic procedure such as a CT-guided TTNA, surgical resection, PET and/or serial CT imaging over a series of months.

Biopsies not yielding a diagnosis of malignant cancer were classified in the patient's chart as negative for lung cancer. The determination of negative for lung cancer was confirmed from negative findings by a second biopsy procedure. Tissue biopsies were taken using TTNA, or surgical resection. When the results of this second more invasive biopsy sample were absent of malignant tissue, the reported finding was benign for malignant lung cancer. Details of the biopsy specimens' medical 
documentation included pathology findings, noting pneumonia, lesions that disappeared on follow-up imaging, and lesions that demonstrated stability over 24 months. Lesions with ground glass changes or inflammation in biopsy reports were further characterized. A false-negative biopsy was defined as biopsies that were originally biopsied, found to be negative for malignancy, yet were later determined to be malignant by percutaneous or surgical biopsy.

\section{- Data analysis}

Continuous variables are expressed using mean, standard deviation and range. Dichotomous variables are summarized as simple proportions. Statistical significance is reported using a $t$-test to evaluate variance between groups.

\section{Results}

In total, 48 patients were able to complete the TV-EXP-mapped EMN procedure and continued to be followed. Two of the patients initially scheduled for the TV-EXP-mapped EMN procedure exhibited excessive coughing after or during the linear EBUS phase of the procedure and therefore they were not exposed to the TV-EXPmapped EMN procedure, thus there leaving no data to report on these patients. The remaining 48 patients had 61 lesions biopsied. Table 3 details the overall prevalence of malignancy found to be $39.6 \%$ (19/48) consistent with prior studies reporting 39.6, 37 and 42.5\% malignancy rates in their high-risk patient populations meeting the lung cancer screening guidelines $[3,12,24]$. Community pulmonologists may expect to experience a similar incidence of positive findings which is well within range as the malignancy rate reported by the NLST, therefore less invasive testing is warranted.

TV-EXP mapping ENB findings report an overall diagnostic yield of $83.3 \%$. 15 nodules measured $10 \mathrm{~mm}$ or smaller, 22 measured 11-20 mm, 14 measured $21-30 \mathrm{~mm}$ and ten lesions were larger than $31 \mathrm{~mm}$ reported in Table 4. LungRADs and Fleishner Society guidelines recommend biopsying nodules $8-20 \mathrm{~mm}$ in size if the patient's risk profile is high risk. Our hospital tumor review board commonly follows these guidelines for patient's that present with a nodule and fit the high-risk profile. All patients evaluated in the study had a positive

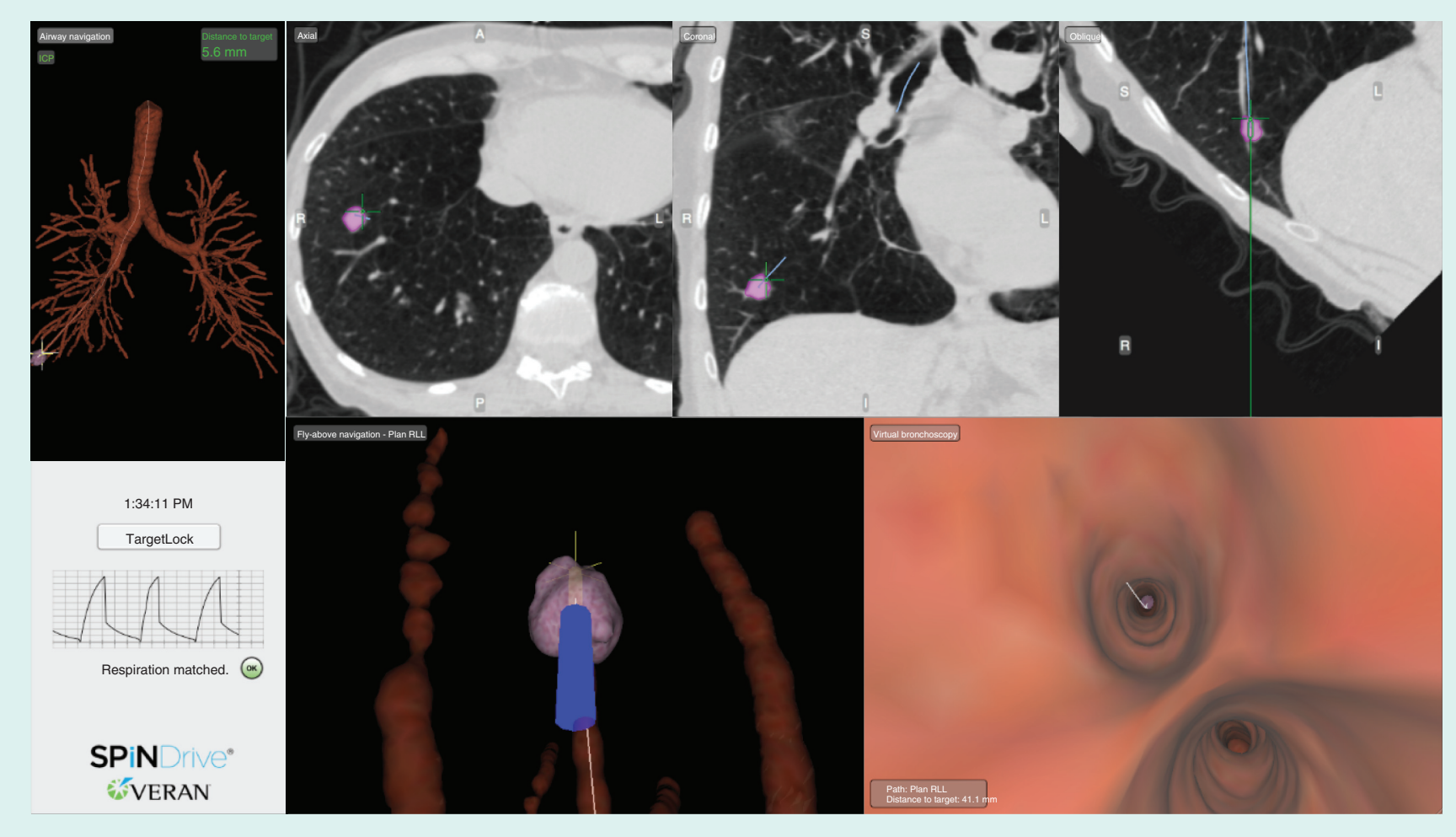

Figure 1. Image of a patient's peripheral pulmonary nodule on the SPiNDrive electromagnetic tip tracked system accessing a positive peripheral lung lesion. 
Table 3. Findings of patient positive for lung cancer.

\begin{tabular}{|lll|}
\hline Cancer & Patients (n) & Cancer detected (\%) \\
\hline Non-small-cell carcinoma & 14 & 74 \\
\hline Small-cell carcinoma & 5 & 26 \\
\hline $\begin{array}{l}\text { Incidence of 39.6\% (19/48) is representative of the lung cancer screening population who are expected to experience a lower } \\
\text { incidence of lung cancer compared with patients with obvious signs and symptoms. Importantly this number of positive findings is } \\
\text { consistent with the reported incidence in the National Lung Screening Trial. }\end{array}$ \\
\hline
\end{tabular}

finding on CT, and a recommendation for lung biopsy by the tumor review board based on patient risk profile. Multiple malignancies for nodules smaller than $20 \mathrm{~mm}$ were diagnosed leading to earlier stage diagnoses as intended by the USPSTF guidelines. Lung lesions were frequently located in the upper lobes, 23 located in the RUL, 16 RLL, three RML, 11 LUL and eight LLL as reported in Table 5.

\section{Discussion of community pulmonologist initial experience}

The TV-EXP mapping EMN procedure took an average of $6.8 \pm 1.5$ samples ranging from four to 11 biopsies. Total procedure times ranged from 35 to 90 min. Pulmonologist TV-EXP mapping planning times ranged from 5 to $10 \mathrm{~min}$. As the pulmonologist gained experience with the system the planning time tended toward $5 \mathrm{~min}$. 40 of $48(83.3 \%)$ patients had a correct diagnosis during their TV-EXP mapping EMN procedure. A correct diagnosis represents a finding that was identical to that of TTNA, watchful waiting, PET or surgical resection. In total, 11 patients were determined to have a malignancy via the procedure obtained biopsy, and 29 patients were determined to be true negative for a malignancy during follow-up with $100 \%$ specificity.

37 patients were initially found to not have a malignancy and were managed using shared decision-making between pulmonologist, patient, hospital tumor review board and their referring physician. The patient data are provided in Table 6.

- Three patients were diagnosed with pneumonia on biopsy;
- Based on PET findings of 12 patients, six patients were referred to TTNA biopsy and one patient was referred to surgical resection. Of the patients referred to PET, four were found to be positive for lung cancer and eight patients were found to be benign;

- Six patients were referred for a PET study revealing limited to no activity. Follow-up CT scanning showed these nodules to be smaller, stable or missing and these patients were determined to be negative for the presence of malignant lung requiring no further intervention at this time;

- In total, 15 were followed up with a serial imaging protocol at 3-6, 9-12 and 18-24 months during the study timeframe. Three of these 15 patients had a nodule that was no longer present during follow-up period. The remaining 12 patients are continuing to be followed with a serial CT or x-ray scan according to recommended guidelines and have shown no signs of nodule growth, all having over 24 months of follow-up. There was one pneumothorax (2\%). No additional complications were reported for the patient group.

In total, 25 of $48(52.1 \%)$ of the patients had a bronchus sign to the lesion. For the patients with a bronchus sign to the lesion, a correct diagnosis was achieved $88 \%(22 / 25)$ of the time. For patients without a bronchus sign to the target, a correct diagnosis was achieved $78.3 \%(18 / 23)$ of the time. This is consistent with reports from prior studies that diagnostic rates are higher for patients with bronchus signs to the lesion [25].

Table 4. Comparison of lesion size, number of lesions and malignancy.

\begin{tabular}{|c|c|c|c|c|}
\hline Size (mm) & Lesions ( $\mathrm{n}$ ) & Benign (n) & Malignant (n) & Malignant (\%) \\
\hline$\leq 10$ & 15 & 14 & 1 & 6.7 \\
\hline$\leq 20$ & 22 & 18 & 4 & 18.2 \\
\hline $21-30$ & 14 & 6 & 8 & 57.1 \\
\hline$>30$ & 10 & 4 & 6 & 60.0 \\
\hline
\end{tabular}


Table 5. Comparison of lung lesion location and malignancy.

\begin{tabular}{lllll|}
\hline $\begin{array}{l}\text { Lung lesion } \\
\text { location }\end{array}$ & Lung lesions $(\mathbf{n})$ & Benign $(\mathbf{n})$ & Malignant $(\mathbf{n})$ & Malignant (\%) \\
RUL & 23 & 13 & 10 & 43.5 \\
RML & 3 & 3 & 0 & 0 \\
RLL & 16 & 14 & 2 & 12.5 \\
LUL & 11 & 5 & 6 & 54.5 \\
\hline LLL & 8 & 7 & 1 & 12.5 \\
\hline LLL: Left lower lobe; LUL Left upper lobe; RLL: Right lower lobe; RML: Right middle lobe; RUL: Right upper lobe. \\
\hline
\end{tabular}

The diagnostic yield for our first 25 patients was $80 \%(20 / 25)$, which we found acceptable and a significant improvement over conventional bronchoscopic techniques using fluoroscopic guidance for these lesions. The diagnostic yield for the next 23 patients was $87 \%$ (20/23) revealing a promising trend toward improvement due to a learning curve with the technology. While the sample size was small, nevertheless the results reflect the experience of one physician faced with the need to provide a diagnostic solution to patients with a positive CT finding having a high risk for lung cancer. Nodule size was fairly consistent across the first and second set of patients (18.4 vs $20.4 \mathrm{~mm}$, respectively). Adapting to the TV-EXP-mapped EMN technique is operator dependent, therefore the learning curve may be different for other users.

Bronchoscopy with guidance has evolved as a viable option however the operator requires expertise in newer guided techniques. Minimally invasive diagnostic procedures using bronchoscopy, ENB, TTNA or TTNB can provide a definitive diagnosis early and therefore may be preferred management strategies for patients meeting the lung cancer screening guidelines. However, balancing the risk of a higher incidence of procedurerelated complications must be balanced with the likelihood of a negative finding; clinical team decision-making is essential in aiding the management of these patients. Early experience with the TV-EXP-mapped EMN offers an additional option reporting positive operator experience and definitively positive diagnostic yield with high ability to rule out lung cancer.

The new challenge facing pulmonologists is diagnosing lung cancer earlier through appropriately applying the less invasive biopsy methodology due to the low rate of positive lung cancer findings. Patients are presenting with smaller lesions and without symptoms. As high-risk patients are screened, identified nodules will be recommended for further workup. Noting the high number of positive CT scan in the NLST, the community pulmonologist is challenged to identify a minimally invasive method to consistently reach these lesions and acquire adequate tissue samples causing little complications leading to increased patient morbidity. The new TV-EXPmapped EMN system, evaluated in this study, shows promise to help lung specialists meet this new challenge. This technique has a low complication profile and a high probability of identifying patients with benign tissue pathology [26].

The hybrid inspiration/expiration CT protocol along with the respiratory gating capabilities of the system seemed to offer an improvement in diagnostic yield when compared with TLCENB with equivalence to R-EBUS and ENB. With the limited space in most bronchoscopy

Table 6. 37 patients follow-up after tidal volume expiration mapping electromagnetic navigation to verify confirmatory diagnosis to aid treatment decisions.

\begin{tabular}{|c|c|c|c|c|}
\hline $\begin{array}{l}\text { Follow-up/ } \\
\text { diagnosis }\end{array}$ & $\begin{array}{l}\text { Diagnostic } \\
\text { method }\end{array}$ & $\begin{array}{l}\text { Lung cancer status } \\
\text { (number) }\end{array}$ & Total patients $(\mathrm{n})$ & $\begin{array}{l}\text { Patients diagnosis } \\
\text { due to follow-up (\%) }\end{array}$ \\
\hline Pneumonia & Biopsy & Benign three & 3 & 8.1 \\
\hline Malignant & TTNA & Malignant seven & 12 & 32.4 \\
\hline Malignant & Surgical resection & Malignant one & 1 & 2.7 \\
\hline $\begin{array}{l}\text { PET - inactive + CT } \\
\text { stable }\end{array}$ & & Benign six & 6 & 16.2 \\
\hline$C T$ - disappeared & & Benign three & 3 & 8.1 \\
\hline $\begin{array}{l}\text { CT stable - } \\
24+\text { months }\end{array}$ & & Benign 12 & 12 & 32.4 \\
\hline
\end{tabular}


suites, the opportunity to not utilize fluoroscopy offers a cost-effective option. Having EM sensors embedded in the actual biopsy instrumentation was easy to use. The embedded EM sensors allowed the pulmonologist to never lose navigation, especially at the most critical time, while performing the biopsy. Using common bronchoscopic tools such as the EM always-on tip tracked forceps in conjunction with a standard bronchoscope made the procedure seamless to learn and to adapt to a procedure routine in the bronchoscopy suite.

\section{Conclusion}

At AnMed (SC, USA), a community-based hospital, this new technology has significantly changed our approach to suspicious PPN. Increased confidence in our ability to accurately reach smaller PPNs has been meaningful in support of our desire to completely address the patient's spectrum of biopsy options. The ability to collect staging samples and to accurately biopsy the lesion in a single procedure is encouraging to streamline patient care. The future of lung cancer diagnosis and management in the early stages will be directly impacted by our ability to embrace new approaches of patient care that enable us to make informed decisions relative to the patient's risk profile to appropriately select the patient's optimum treatment algorithm. Additional studies of interest would be to further examine the comparison of different registration methods offered by the navigation systems. With accuracy being of vital importance in navigation procedures, it would be interesting to exam target location on the navigation system for the same patient with different registration techniques that advance the TV-EXP-mapped EMN procedure.

The aim of this study was to determine the community pulmonologist learning curve, use, safety and diagnostic yield of the TV-EXP mapping EMN procedure to enable access of EM forceps to access commonly found lung lesions in a CT screened patient population. Our initial findings suggest this approach shows potential to improve the diagnostic yield of transbronchial lung biopsy.

Financial \& competing interests disclosure

$L$ Amir, MS, MHA received a small stipend from Veran Medical Technologies. The authors have no other relevant affliations or financial involvement with any organization or entity with a financial interest in or financial conflict with the subject matter or materials discussed in the manuscript apart from those disclosed.

No writing assistance was utilized in the production of this manuscript.

\section{Ethical conduct of research}

The authors state that they have obtained appropriate institutional review board approval or have followed the principles outlined in the Declaration of Helsinki for all human or animal experimental investigations. In addition, for investigations involving human subjects, informed consent has been obtained from the participants involved.

\section{Open access}

This work is licensed under the AttributionNonCommercial-NoDerivatives 4.0 Unported License. To view a copy of this license, visit http://creativecommons.org/ licenses/by-nc-nd/4.0/

\section{References}

1 American Cancer Society website. www.cancer.org

2 Moyer VA. U.S. Preventive Services Task Force. Screening for lung cancer: U.S. Preventive Services Task Force recommendation statement. Ann. Intern. Med. 160(5), 330-338 (2014).

3 The National Lung Screening Trial Research Team; Aberle DR, Adams AM, Berg CD et al. Reduced lung-cancer mortality with low-dose computed tomographic screening. N. Engl. J. Med. 365, 395-409 (2011).

4 Ost DE, Gould MK. Decision making in patients with pulmonary nodules. Am. J. Respir. Crit. Care Med. 185(4), 363-372 (2012).
5 Welker JA, Alattar M, Gautam S. Repeat needle biopsies combined with clinical observation are safe and accurate in the management of a solitary pulmonary nodule. Cancer 103(3), 599-607 (2005).

6 Gould MK, Fletcher J, Iannettoni MD et al. Evaluation of patients with pulmonary nodules: when is it lung cancer? ACCP evidence-based clinical practice guidelines (2nd edition American College of Chest Physicians). Chest 132(Suppl. 3), S108-S130 (2007).

7 Rivera MP, Mehta AC, Wahidi MM. Establishing the diagnosis of lung cancer: diagnosis and management of lung cancer, 3rd ed; American College of Chest Physicians evidence-based clinical practice guidelines. Chest 143(5 Suppl.), e142S-e165S (2013).
8 Silvestri GA, Feller-Kopman D, Chen A et al. Latest advances in advanced diagnostic and therapeutic pulmonary procedures. Chest 142 , 1636-1644 (2012).

9 Memoli JSW, Nietert PJ, Silvestri GA. Meta-analysis of guided bronchoscopy for the evaluation of the pulmonary nodule. Chest 142(2), 385-393 (2012).

10 Schreiber G, McCrory DC. Performance characteristics of different modalities for diagnosis of suspected lung cancer: summary of published evidence. Chest 123, S115-S128S (2003).

11 Baaklini WA, Reinoso MA, Gorin AB, Sharafkaneh A, Manian P. Diagnostic yield of fiberoptic bronchoscopy in evaluating solitary pulmonary nodules. Chest 117(4), 1049-1054 (2000). 
12 Seijo LM, de Torres JP, Lozano MD et al. Diagnostic yield of electromagnetic navigation bronchoscopy is highly dependent on the presence of a bronchus sign on CT imaging: results from a prospective study. Chest 138(6), 1316-1321 (2010).

13 Lee KA, Raval AA, Amir L. Cost-effectiveness of endobronchial percutaneous biopsy compared with transthoracic biopsy for diagnosis of peripheral lung lesions. Lung Cancer Manag. 3(2), 135-148 (2014).

14 Eberhardt R, Anantham D, Ernst A, Feller-Kopman D, Herth F. Multimodality bronchoscopic diagnosis of peripheral lung lesions. Am. J. Respir. Crit. Care Med. 176, 36-41 (2007).

15 Nabavizadeh N, Zhand J, Elliott DA et al. Electromagnetic navigational bronchoscopyguided fiducial markers for lung stereotactic body radiation therapy. J. Bronchol. Intervent. Pulmonol. 21, 123-130 (2014).

16 Becker HC, Herth F, Ernst A, Schwarz Y. Bronchoscopic biopsy of peripheral lung lesions under electromagnetic guidance: a pilot study. J. Bronchoscopy 12, 9-13 (2005).
17 Schwarz Y, Greif, , Becker HD, Ernst A, Mehta A. Real-time electromagnetic navigation bronchoscopy to peripheral lung lesions using overlaid CT images: the first human study. Chest 129, 988-994 (2006).

18 Gildea TR, Mazzone PJ, Karnak D, Meziane $\mathrm{M}$, Mehta AC. Electromagnetic navigation diagnostic bronchoscopy: a prospective study. Am. Respir. Crit. Care Med. 174, 982-989 (2006).

19 Wahidi MM, Herth F, Yasufuku K et al. Technical aspects of endobronchial ultrasound-guided transbronchial needle aspiration CHEST Guideline and Expert Panel Report. Chest 149(3), 816-835 (2016).

20 Gex G, Pralong JA, Combescure C et al. Diagnostic yield and safety of electromagnetic navigation bronchoscopy for lung nodules: a systematic review and meta-analysis. Respiration 87, 165-176 (2014).

21 Bauer M, Joshiy S, Modinz K. Diffeomorphic density matching by optimal information transport. Cornell University Library (2015). http://arxiv.org/pdf/1501.07635.pdf
22 Chen A, Pastis N, Furukawa B, Silvestri G. Effect of respiratory motion on pulmonary nodule location during electromagnetic navigation bronchoscopy. Chest 147(5), 1275-1281 (2014).

23 Appelbaum L, Sosna J, Nissenbaum Y, Benshtein A, Goldberg SN. Electromagnetic navigation system for CT-guided biopsy of small lesions. AJR Am. J. Roentgenol. 106, 1194-1200 (2011).

24 Mehta H, Ravenel J, Shaftman S et al. The utility of nodule volume in the context of malignancy prediction for small pulmonary nodules. Chest 145(3), 464-472 (2014).

25 Lacunza MM, Zulueta JJ. Diagnostic yield of electromagnetic navigation bronchoscopy is highly dependent on the presence of a Bronchus sign on CT imaging: results from a prospective study. Chest 138, 1316-1321 (2010).

26 Gilbert C, Akulian J, Ortizo R et al. Novel bronchoscopic strategies for the diagnosis of peripheral lung lesions: present techniques and future directions. Respirology 19(5), 636-644 (2014). 